\title{
TEM Characterization of GaSb growth on GaAs (001) Substrate: Growth Mode and Defect Evaluation
}

\author{
Shenghong Huang*, Ganesh Balakrishnan**, and Diana L. Huffaker*** \\ * Department of Earth and Planetary Sciences, University of New Mexico, Albuquerque, NM 87131 \\ ** Center for High Technology Materials, University of New Mexico, Albuquerque, NM 87106 \\ *** California Nano Systems Institute and Department of Electrical Engineering, University of \\ California, Los Angeles, CA 90095
}

Exploiting extreme lattice-mismatch of Sb-based epitaxy on GaAs and Si substrates are of considerable interest for III-Sb optoelectronic devices on GaAs substrate such as monolithically integrated lasers, detectors, solar cells and transistors. Recent technical advancements in GaSb substrates have enabled high quality lattice-matched GaSb epitaxy on native substrates, but GaAs substrates are desirable for many applications.

Due to $7.8 \%$ lattice mismatch between GaSb epilayer and GaAs substrate, standard approaches involve tetragonal distortion in which the material relieves strain energy through $60^{\circ}$ misfits and threading dislocations. The vertically propagating defects associated with this growth mode will often jeopardize both their electrical and optical properties of the device structures with nonradiative recombination and carrier leakage.

During the interfacial misfit (IMF) growth mode, the initial strain relaxation of highly mismatched GaSb layers grown on GaAs (001) is governed by the two-dimensional (2D), periodic interfacial misfit (IMF) dislocation array growth mode. Under optimized growth conditions, only pure $90^{\circ}$ dislocations are generated along both [110] and [1-10] directions that are located at GaSb/GaAs interface, which leads to very low threading dislocation density propagated along the growth direction. Our experimental results show that this interaction causes $\mathrm{Sb}$ to participate in the formation of a periodic IMF array instead of simply giving rise to tetragonal distortion in the evolving GaSb layer.

We through TEM technique analyze the defect formation and measure their dislocation density for IMF growth mode and non-IMF growth mode. The long-range uniformity and subsequent strain relaxation of the 2D and periodic IMF array are demonstrated via transmission electron microscopy and scanning transmission electron microscopy images at GaSb/GaAs interface.

The low-dislocation density, strain-relieved GaSb layers formed by the IMF growth conditions have been demonstrated to be applied in the monolithic integration of semiconductor devices.

References:

[1] S. H. Huang et al., Appl. Phys. Lett., 88 (2006) 131911.

[2] S. H. Huang et al., J. Appl. Phys., 105 (2009) 103104. 


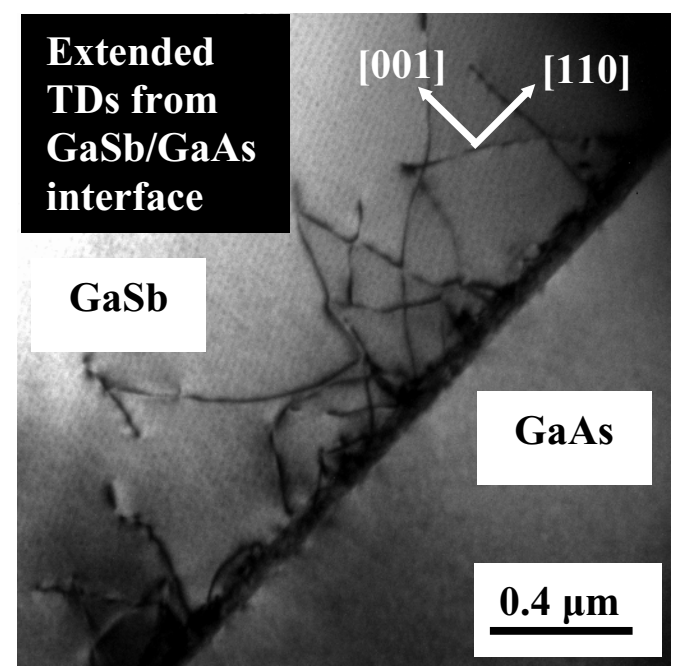

Figure 1: Bright-field cross-sectional TEM images (zone axis [1-10]) of GaSb grown on GaAs under non-IMF growth mode, indicating high threading dislocations are extended from $\mathrm{GaSb}$ /GaAs interface.
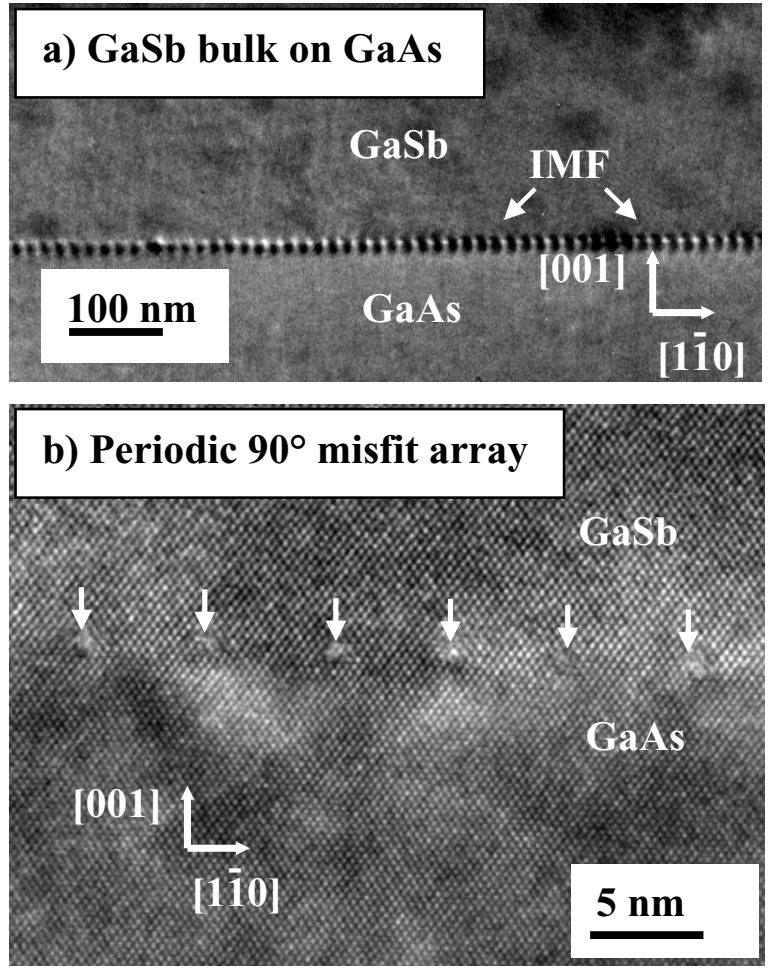

Figure 2: Cross sectional TEM image (zone axis [110]) of GaSb on GaAs under IMF growth mode, a) low magnification, showing a highly periodic array of misfit dislocations at the $\mathrm{GaSb} / \mathrm{GaAs}$ interface, b) highmagnification, indicating the misfit separation, measured to be $5.6 \mathrm{~nm}$, corresponds to exactly $13 \mathrm{GaSb}$ lattice sites grown on $14 \mathrm{GaAs}$ lattice sites.
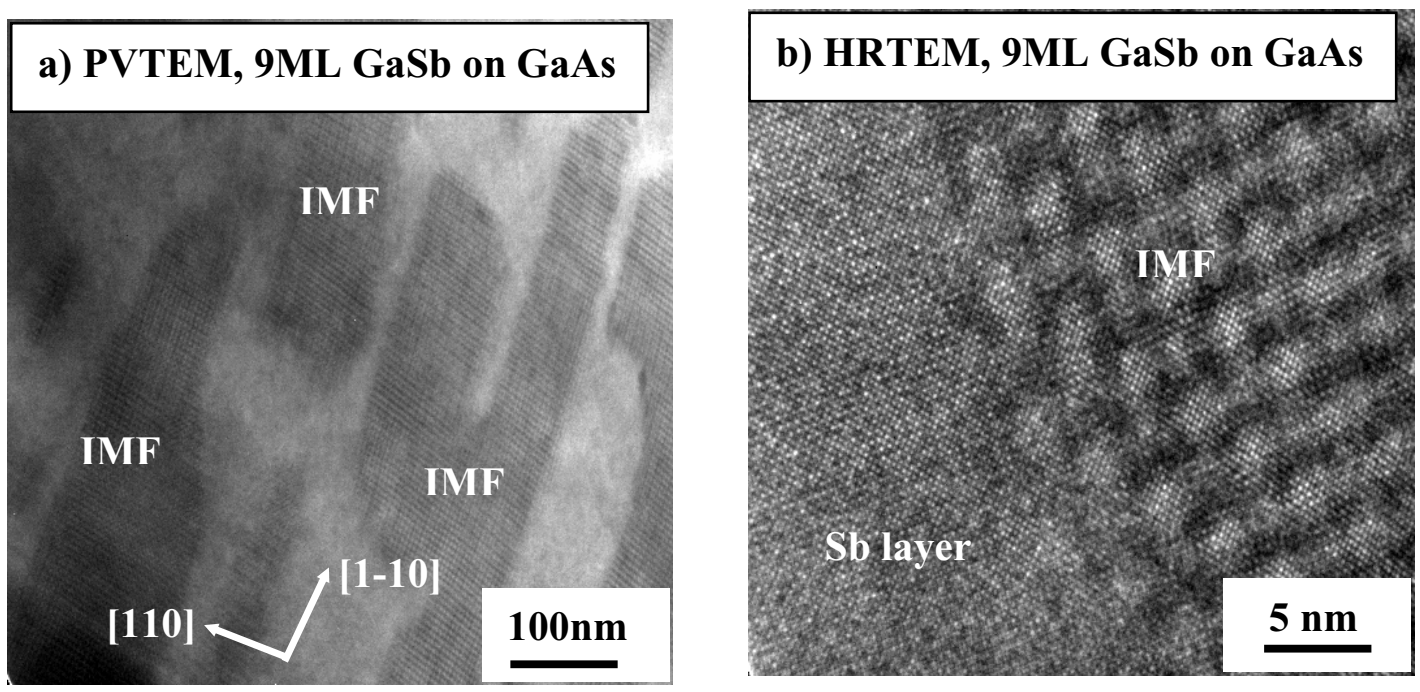

Figure 3: Plan-view bright-field TEM images of $9 \mathrm{ML}$ GaSb grown on GaAs under IMF growth mode, (a) low-resolution image, (b) high-resolution image. 